\title{
Quantum Monte Carlo study of the three-dimensional spin-polarized homogeneous electron gas
}

\author{
G. G. Spink and R. J. Needs \\ TCM Group, Cavendish Laboratory, University of Cambridge, \\ J. J. Thomson Avenue, Cambridge CB3 OHE, United Kingdom \\ N. D. Drummond \\ Department of Physics, Lancaster University, Lancaster LA1 4YB, United Kingdom
}

(Dated: July 23, 2013)

\begin{abstract}
We have studied the spin-polarized three-dimensional homogeneous electron gas using the diffusion quantum Monte Carlo method, with trial wave functions including backflow and three-body correlations in the Jastrow factor, and we have used twist averaging to reduce finite-size effects. Calculations of the pair correlation function, including the on-top pair density, as well as the structure factor and the total energy, are reported for systems of 118 electrons in the density range $r_{\mathrm{s}}=0.5-20$ a.u., and for spin polarizations of $0,0.34,0.66$, and 1 . We consider the spin resolution of the pair correlation function and structure factor, and the energy of spin polarization. We show that a control variate method can reduce the variance when twist-averaging, and we have achieved higher accuracy and lower noise than earlier quantum Monte Carlo studies.

PACS numbers: 71.10.Ca, 02.70.Ss, 71.15.Nc, 67.10.-j
\end{abstract}

\section{INTRODUCTION}

The simplicity of the three-dimensional (3D) homogeneous electron gas (HEG) model,,$\frac{1}{\text { consisting of electrons }}$ interacting via the Coulomb potential in a uniform, positive background for charge neutrality, allows the study of important features of the many-electron problem without the complication of a lattice potential. Early pioneers of quantum mechanics discovered much of the phenomenology of the HEG, including Wigner's celebrated insight ${ }^{2.3}$ that the fluid will crystallize at low density. More recently, the continuing research effort to understand the behavior of the HEG is motivated by the model's application in density functional theory (DFT).

Quantum Monte Carlo (QMC) methods can provide accurate estimates of the static properties of interacting many-body systems such as the HEG. The ground state of the 3D HEG for collinear spins is accepted to be an unpolarized Fermi fluid at high densities. As the density decreases, QMC calculations indicate an apparently continuous transition to a spin-polarized fluid occurs, starting at a density of about $r_{\mathrm{s}}=50 \pm 2 \mathrm{a} \cdot \mathrm{u} . \underline{\underline{4}}$ The transition to a Wigner crystal is calculated to occur at about $r_{\mathrm{s}}=106 \pm 1$ a.u. $\stackrel{5}{=}$ QMC calculations have furnished data used within DFT, most notably the energies of the HEG calculated by Ceperley and Alder,$\underline{3}$ which are used to parameterize the local-density-approximation exchange-correlation functional. Semilocal $\underline{6}^{6}$ and nonlocal $1^{7.8}$ exchange-correlation functionals may use additional properties of the HEG such as the pair correlation function (PCF) and, especially, the on-top pair density ${ }^{9}$

The PCF is a measure of the spatial correlations of electron positions that arise from the Coulomb repulsion and Pauli exclusion. The spin-resolved PCF of the HEG, $g_{\alpha \beta}(r)$, is defined such that $n_{\beta} g_{\alpha \beta}(r) 4 \pi r^{2} d r$ is the expected number of spin- $\beta$ electrons in an infinites- imal shell of radius $r$ when a spin- $\alpha$ electron is found at the origin and $n_{\beta}$ is the number density of spin- $\beta$ electrons. Defining the spin polarization $\zeta$ as the ratio $\zeta=\left(N_{\uparrow}-N_{\downarrow}\right) /\left(N_{\uparrow}+N_{\downarrow}\right)$, the total PCF $g$ is a weighted average of the spin-resolved components:

$$
g=\left(\frac{1+\zeta}{2}\right)^{2} g_{\uparrow \uparrow}+\left(\frac{1-\zeta}{2}\right)^{2} g_{\downarrow \downarrow}+\left(\frac{1-\zeta^{2}}{2}\right) g_{\uparrow \downarrow} .
$$

The on-top pair density $g(0)$ is the value of the PCF at contact. The region around $r=0$ is, however, the most challenging to sample in a stochastic simulation, particularly at low densities, as the electrons are rarely found close to one another. Toulouse et al $\underline{\underline{10}}$ have developed a possible solution to this difficulty, extending the zerovariance zero-bias estimators of Assaraf and Caffarel $\stackrel{11}{\underline{1 t}}$ It has recently been found by Fantoni, $\frac{12}{2}$ however, that the zero-bias correction required in DMC calculations significantly increases the variance. We therefore opted to use the traditional histogram estimator of the $\mathrm{PCF}$ in this work; our simulations gathered enough data to ensure good precision in the reported $g(0)$ values.

In light of the above challenges, while QMC methods have been very successful in calculating many quantities of interest in the HEG, such as the energy and the PCF at intermediate distances, the short-range behavior of the PCF has usually been obtained by fitting QMC data to some analytical function, which can be constrained to obey exact results such as the electron-electron cusp conditions. Even then, however, there is some disagreement between the PCFs obtained using different approaches. Gori-Giorgi and Perdew developed an analytical model based on exact constraints and QMC energy data. $\underline{13,14}^{14}$ The same authors $\frac{15}{15}$ extended an approach originally due to Overhauser ${ }^{16}$ using a screened Coulomb potential and two-electron wave functions. An alternative starting point is afforded by ladder theory, which gave rise to an 
early and widely used result for $g(0)$ due to Yasuhara 17 Nagy et al $\underline{18}$ offer insight into the good agreement between these results. More recently, Qian $\frac{19}{9}$ was able to relax an approximation made in the earlier ladder theory calculations and obtained a markedly different result. Although several QMC calculations of PCFs for the unpolarized 3D HEG have been reported, less attention has been paid to fully and, especially, partially spin-polarized systems $\underline{12,20-25}$

In this paper we present extensive results for PCFs, static structure factors (SSFs), and energies for HEGs containing 118 electrons over the density range relevant to DFT calculations, $r_{\mathrm{s}}=0.5-20$ a.u. We consider four values of the spin polarization, $\zeta=0,40 / 118,78 / 118$, and 1 , although in the text we refer to the intermediate polarizations as $0.34(40 / 118)$ and $0.66(78 / 118)$. We use Slater-Jastrow wave functions incorporating backflow and three-body correlations, we investigate twistaveraged boundary conditions and finite-size effects, and we are able to gather sufficient data to ensure that the statistical uncertainty is relatively modest. A variance reduction method is found to improve significantly the precision of twist-averaged data without introducing bias. We show the spin resolution of the PCFs and the SSFs, and we consider in particular the on-top pair density and the energy of spin polarization as functions of $r_{\mathrm{s}}$ and $\zeta$. We have performed spline fits to our PCF data, which appear to be more accurate than polynomial fits. A short Fortran 90 program is available to evaluate the spline fits to our PCF data $\underline{26}$

Our paper is organized as follows. In Sec. I we discuss the QMC methods used in our calculations. Section III describes our PCF, SSF, and energy data and shows comparisons to results in the existing literature. We offer our conclusions in Sec. IV Hartree atomic units are used throughout this paper, so that $\hbar=|e|=m_{e}=4 \pi \epsilon_{0}=1$.

\section{METHODS}

We have used the CASINO ${ }^{27}$ code to perform variational and diffusion Monte Carlo 28 (VMC and DMC) calculations. In the VMC method, the Metropolis algorithm is used to generate a set of configurations distributed according to the square modulus of a trial wave function over which the local energy is averaged. In the DMC method, an initial wave function is evolved in imaginary time, which in principle projects out the ground state. For fermionic systems, the antisymmetry of the wave function is, however, imposed via the fixed-node approximation,$\frac{29}{9}$ in which the nodal surface is constrained to remain unchanged during the evolution. Both the VMC and DMC methods give an upper bound to the ground-state energy of the system. The quality of the parameterization of the trial wave function and the VMC optimization procedure influence the accuracy of the results and the statistical noise.

Our wave functions consisted of Slater determinants of plane waves multiplied by a Jastrow factor and included a backflow transformation. The Jastrow factor comprised polynomial and plane-wave expansions in the electronelectron separation, together with three-body terms $\underline{30,31}$ The electron coordinates in the Slater determinant were replaced by quasiparticle coordinates obtained by a backflow transformation represented by a polynomial in the electron-electron separation $\stackrel{32.33}{=}$ The variable parameters in these wave functions were optimized using variance minimization ${ }^{34,35}$ and then linear least-squares energy minimization, $\underline{36}$ as this was found to give the most accurate trial wave functions.

The quality of our wave functions was such that, for unpolarized and fully polarized systems, the VMC and DMC methods produced essentially identical PCFs within the statistical precision we were able to obtain. For these systems we therefore report DMC expectation values rather than the extrapolated estimators often used in QMC studies. In partially polarized systems, however, our trial wave functions led to small but statistically significant differences between the VMC and DMC data for minority-spin electrons. In these cases we have used extrapolated estimation, $\sqrt[37]{=}$ in which the PCF is $g=2 g_{\mathrm{DMC}}-g_{\mathrm{VMC}}$, where $g_{\mathrm{DMC}}$ and $g_{\mathrm{VMC}}$ are the DMC and VMC PCFs, respectively.

We studied 118-electron HEGs in face-centeredcubic simulation cells and imposed twisted boundary conditions ${ }^{38}$ so that the wave function picks up a phase when an electron is translated by a simulation-cell lattice vector $\mathbf{R}_{s}$ :

$\Psi\left(\mathbf{r}_{1}, \ldots, \mathbf{r}_{i}+\mathbf{R}_{s}, \ldots, \mathbf{r}_{N}\right)=\exp \left(i \mathbf{k}_{s} \cdot \mathbf{R}_{s}\right) \Psi\left(\mathbf{r}_{1}, \ldots, \mathbf{r}_{N}\right)$.

The twist offset $\mathbf{k}_{s}$ was allowed to vary with uniform probability over the first Brillouin zone of the simulation cell during our simulations. Averaging in this way has been shown to reduce single-particle finite-size effects in the energy 39

We investigated the remaining variation with system size in the PCF using twist-averaged VMC simulations at $r_{\mathrm{s}}=5$ a.u. and $\zeta=0$. We show the spin resolved PCF in Fig. 1. This figure, like all others in this paper except Fig. 12, shows our raw QMC data, rather than a fit to the data. The region around the first peak in the antiparallel-spin PCF best shows the variation with system size. Both $g_{\uparrow \downarrow}(r)$ (upper curves) and $g_{\uparrow \uparrow}(r)$ (lowerright curves) exhibit systematic finite-size errors for small systems in this region, converging to the thermodynamic limit on the scale of the graph at approximately $N=100$ electrons. (Results for 118- and 226-electron systems are almost indistinguishable on the scale of the graph.) Some cancellation of errors occurs when the spin-averaged PCF is calculated.

The variation with system size appears less pronounced and less systematic at smaller $r$. The on-top pair density was unaffected within statistical uncertainty. For systems with $N=54,118$, and 226 electrons, there was good agreement at small but nonzero distances: typical differences were of order $1-5 \%$. In contrast, the long-range 


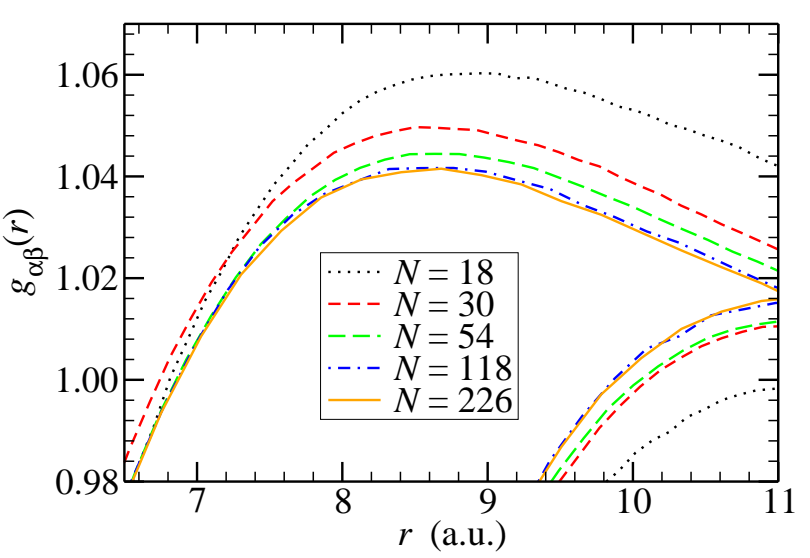

FIG. 1. (Color online) Finite-size effects in the spin-resolved pair correlation function (PCF). The upper curves are for antiparallel spins and the lower-right curves are for parallel spins. The data are from VMC twist-averaged simulations at $r_{\mathrm{s}}=5$ a.u. and $\zeta=0$, and at the various sizes shown. The region around the first peak in the antiparallel-spin PCF is shown, where the largest finite-size effects occur.

PCF is affected by unavoidable finite-size errors due to the finite size of the simulation cell. Twist-averaging cannot remove the spurious correlation caused by the periodic boundary conditions. This shows up as a small but statistically significant variation in the long-range oscillations from about $r / r_{s} \gtrsim 2$ for 118 electrons that decreases in magnitude as the system size increases. Figure 1 is included as an XMGrace file in the Supplemental Material 26 accompanying this paper, so that other regions of the graph may be inspected.

The energy is known to converge much more slowly with system size than the $\mathrm{PCF}, \underline{38,39}$ We have therefore applied analytical corrections to our energy data following the procedure developed in Refs. 40 and 39 . Residual single-particle finite-size effects are removed by addition of the difference between the infinite-system and twist-averaged, finite-system Hartree-Fock kinetic energies. The leading-order correction to the Ewald energy, and leading- and next-to-leading-order corrections to the kinetic energy, are also included. In addition, finite-size effects in the energy have previously 25 been found to be relatively insensitive to $\zeta$, and so a cancellation of errors renders finite-size effects in the energies of spin polarization small even without any correction. The analytical arguments of Holzmann et al $\underline{\underline{41}}$ lend further support to this conclusion.

We also examined possible time-step and populationcontrol biases and found them to be very small-less than one part in a thousand of the correlation energy in test cases - so we neglect them in what follows. All DMC calculations were performed using at least 1000 walkers and time steps for which the acceptance probability was generally greater than $99.7 \%$.

We accumulate the spin-resolved $\mathrm{PCF}$ as an average over the set of configurations generated in the course of the simulation:

$$
g_{\alpha \beta}(r)=\frac{\Omega}{4 \pi r^{2} N_{\alpha} N_{\beta}}\left\langle\sum_{j \in \alpha} \sum_{\substack{l \in \beta \\ l \neq j}} \delta\left(\left|\mathbf{r}_{j}-\mathbf{r}_{l}\right|-r\right)\right\rangle,
$$

where $\Omega$ is the simulation-cell volume and $N_{\alpha}$ is the number of electrons of spin $\alpha$. The SSF is simply related to the Fourier transform of the PCF and is given by

$$
S_{\alpha \beta}(\mathbf{k})=\frac{1}{\sqrt{N_{\alpha} N_{\beta}}}\left\langle\rho_{\alpha}(\mathbf{k}) \rho_{\beta}(-\mathbf{k})\right\rangle-\sqrt{N_{\alpha} N_{\beta}} \delta_{\mathbf{k} \mathbf{0}}
$$

where $\rho_{\alpha}(\mathbf{k})=\sum_{j \in \alpha} \exp \left(i \mathbf{k} \cdot \mathbf{r}_{j}\right) . S_{\alpha \beta}(\mathbf{k})$ is then spherically averaged in k-space, because the SSF only depends on the magnitude of $\mathbf{k}$ in a homogeneous and isotropic system.

At high densities, the variation in the energy as the twist offset is changed can be much greater than other sources of noise in the simulation. Twist-averaging can therefore be computationally expensive for high-density HEGs, because a large number of twist angles are required in order to obtain a precise energy and the simulation must be reequilibrated between twists, preventing rapid changes of twist offset in a DMC calculation. To circumvent this difficulty, we have used the HartreeFock energy (as a function of twist offset) as a control variate. $\underline{\underline{42}}$

If $E_{\mathrm{QMC}}$ denotes the QMC energy, $E_{\mathrm{HFKE}}$ the HartreeFock kinetic energy, and $E_{\mathrm{HFEx}}$ the exchange energy as functions of twist angle, the standard way of getting the twist-averaged energy is $\left\langle E_{\mathrm{QMC}}\right\rangle$, where the angled brackets denote an average over twists. Another estimator is

$\theta=E_{\mathrm{QMC}}+c_{1}\left(E_{\mathrm{HFKE}}-\left\langle E_{\mathrm{HFKE}}\right\rangle\right)+c_{2}\left(E_{\mathrm{HFEX}}-\left\langle E_{\mathrm{HFEX}}\right\rangle\right)$,

where the $\left\{c_{i}\right\}$ are coefficients that can be chosen to minimize the variance of $\theta$. In order to account for the remaining sources of noise, we add the correction above to the energy data at each time step and, at the end of the simulation, reblock ${ }^{43}$ the corrected data to obtain much smaller error bars.

To illustrate the reduction in variance obtained, we apply the method to VMC data for two systems in this section: first a HEG with two up-spin electrons and one down-spin electron at $r_{\mathrm{s}}=0.5$ a.u.; then a 118 -electron HEG at $r_{\mathrm{s}}=0.5$ a.u. and $\zeta=0$. The VMC method allows one to simulate a large number of twist angles in parallel, so we are able to compare the corrected data to accurate twist-averages performed the standard way. It can be seen in Tables \and $\Pi$ that the method successfully reduces the variance without introducing bias. Although we chose twist offsets randomly throughout the first Brillouin zone, the method might also improve convergence of twist-averages using Monkhorst-Pack ${ }^{44}$ grids. 
TABLE I. Energies from VMC simulations in a HEG at $r_{\mathrm{s}}=$ 0.5 a.u. containing two up-spin electrons and one down-spin electron. The raw VMC energies are compared to the same data after a correction has been applied using HF kinetic and exchange energies as described in the text. The VMC twistaveraged energy obtained in the standard way, using just over $21 \times 10^{6}$ twist angles, is 3.5484(3) a.u. per electron.

\begin{tabular}{lll}
\hline \hline \multirow{2}{*}{ twists } & \multicolumn{2}{c}{ VMC energy (a.u./elec.) } \\
Raw & Corr. \\
\hline 10 & $3.73(40)$ & $3.5480(1)$ \\
20 & $3.57(39)$ & $3.5480(1)$ \\
100 & $3.42(10)$ & $3.5480(1)$ \\
441 & $3.51(6)$ & $3.5480(1)$ \\
\hline \hline
\end{tabular}

TABLE II. Energies from VMC simulations in a 118-electron HEG at $r_{\mathrm{s}}=0.5$ a.u. and $\zeta=0$. The raw VMC energies are compared to the same data after a correction has been applied using HF kinetic and exchange energies as described in the text. The VMC twist-averaged energy obtained in the standard way, using $4.8 \times 10^{6}$ twist angles, is $3.41378(2)$ a.u. per electron.

\begin{tabular}{lcc}
\hline \hline \multirow{2}{*}{ \# twists } & \multicolumn{2}{c}{ VMC energy (a.u./elec.) } \\
& Raw & Corr. \\
\hline 10 & $3.424(4)$ & $3.41377(4)$ \\
20 & $3.424(4)$ & $3.41375(4)$ \\
50 & $3.414(5)$ & $3.41380(3)$ \\
156 & $3.416(2)$ & $3.41377(2)$ \\
\hline \hline
\end{tabular}

\section{RESULTS}

\section{A. Pair Correlation Function}

Spin-resolved pair correlation functions (PCFs) are shown in Fig. 2 for the unpolarized HEG at different densities using twist averaging. Antiparallel-spin PCFs are translated upwards by 0.2 units for greater visibility, and show much greater variation with density. For $\zeta=0$ (and $\zeta=1$, not shown) the $\mathrm{VMC}$ and DMC results are very similar, so in the plot we present only DMC data.

We show the variation of the PCFs with spin polarization at $r_{\mathrm{s}}=3 \mathrm{a} . \mathrm{u}$. in Fig. 3. In this figure, we translate the antiparallel-spin PCFs upwards by 0.4 units and the parallel-majority-spin PCFs upwards by 0.2 units. For $\zeta=0$ and $\zeta=1$, we have used the DMC values. For $\zeta=0.34$ and $\zeta=0.66$, the extrapolated estimate is shown, because small but statistically significant differences emerged between the DMC and VMC data in the parallel-minority-spin PCFs. Compared with the parallel-spin PCFs, the antiparallel-spin PCF is relatively insensitive to the degree of spin polarization.

The PCF data discussed above are twist-averaged to reduce single-particle finite-size effects. Twist averag-

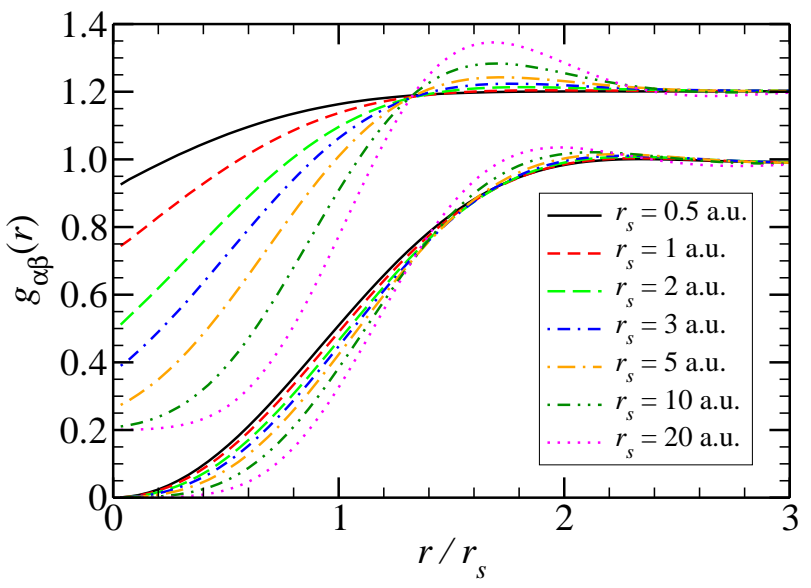

FIG. 2. (Color online) Spin-resolved pair correlation functions (PCFs) for unpolarized HEGs calculated at the densities shown. The antiparallel-spin PCFs are translated upwards by 0.2 units. The data were obtained using twist-averaged DMC simulations.

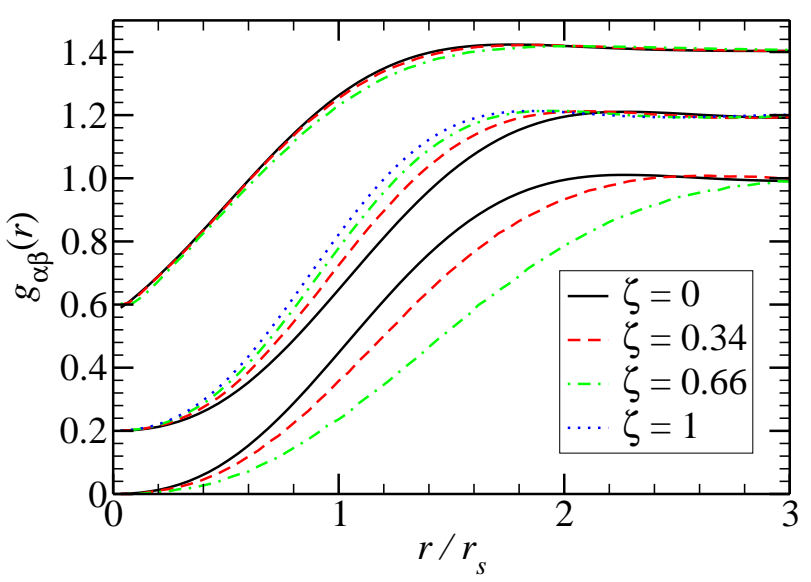

FIG. 3. (Color online) Variation in the pair correlation function (PCF) with spin polarization at $r_{\mathrm{s}}=3$ a.u. Antiparallelspin PCFs are translated upwards by 0.4 units, parallelmajority-spin PCFs are translated upwards by 0.2 units, and the lower curves give the parallel-minority-spin PCFs. Note that the $\zeta=0$ parallel-spin PCF is shown twice (in black) for comparison with both up-up and down-down spin PCFs in those cases where they differ. The DMC data are shown for unpolarized and fully polarized systems, while extrapolated estimates are used for intermediate polarizations. All of the data are twist-averaged.

ing has a significant effect on the energy data, but the effect on the PCF data is much smaller. We show in Fig. 4 the difference $g_{\mathrm{TA}}-g_{\mathrm{PBC}}$ between imposing twistaveraged and periodic boundary conditions for parallel spins. Noise obscures any clear pattern for the antiparallel spins, although there is a tendency for the equivalent figure to show a small dip before rising to zero, so that $g(0)$ appears to be largely unaffected.

We compare our results at $r_{\mathrm{s}}=5$ a.u. and $\zeta=0$ with 


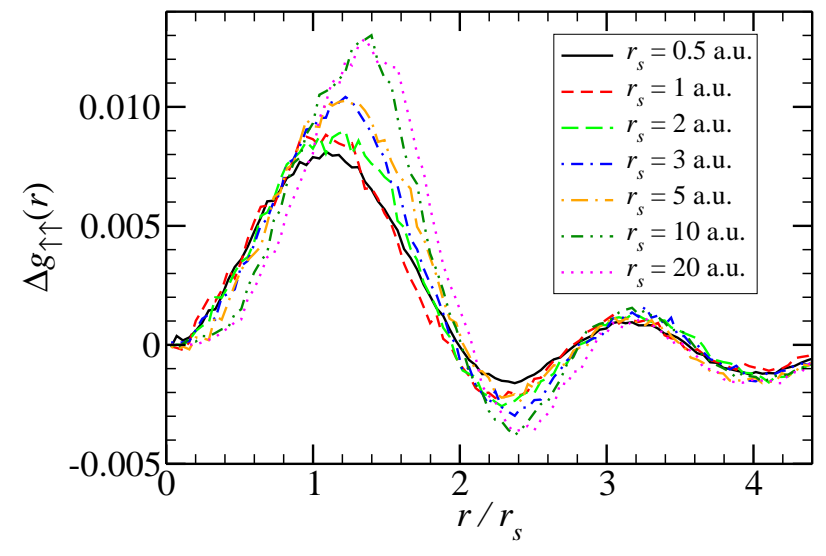

FIG. 4. (Color online) Changes in the pair correlation function (PCF) due to twist averaging. We plot parallel-spin PCFs with twist averaging minus the same quantity evaluated without twist averaging, for the densities shown and $\zeta=0$. Only DMC results are shown, as the VMC results are very similar.

QMC data from the literature in Fig. 5 . We find good agreement with the data of Gori-Giorgi et $a l^{23}$ for intermediate $r / r_{\mathrm{s}}$. Our on-top pair densities are closer to those of Ortiz and Ballone ${ }^{20}$ as shown in the inset, although at intermediate $r / r_{\mathrm{s}}$ modest (of order $5 \%$ ) differences are obtained. The figure shows our raw QMC data, whereas the other curves are fits to the QMC data

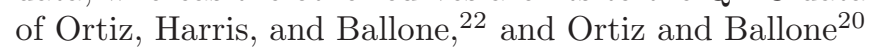
respectively. Both of these fits obey the Kimball cusp conditions and we are confident our QMC data are also consistent with these conditions. As mentioned below, our short-range PCFs are well-described by a quadratic of the form $g_{\uparrow \downarrow}(r)=a+a r+b r^{2}$, which satisfies the first Kimball condition.

We show in Fig. 6 our $g(0)$ values for the unpolarized system in comparison to those in the literature. We have averaged our DMC and VMC data in this figure to reduce statistical uncertainty, because the $\zeta=0$ PCFs produced by the two methods are identical within the statistical precision we were able to obtain. We used the above quadratic form to estimate $g(0)$ from our PCF data at finite distances, $g(r)$, and obtained good fits for all systems. Our results are in good agreement with the recent QMC data of Holzmann et al.,$\stackrel{24}{\underline{4}}$ and the results of GoriGiorgi and Perdew $\underline{\underline{13}}$ who used various data including QMC results, and the results of Yasuhara 17 Our calculations give significantly smaller values of $g(0)$ than those of Gori-Giorgi, Sacchetti, and Bachelet, $\stackrel{23}{2}$ and those of Qian $\stackrel{19}{19}$ This may suggest that Yasuhara's scheme successfully incorporates screening effects into the ladder theory calculations. Nagy et al $\stackrel{18}{\underline{18}}$ suggest that the description of screening used by Gori-Giorgi and Perdew is also an important factor in explaining the success of the latter authors' model.

We have also obtained on-top pair densities for partially polarized systems; see Fig. 7. In this case we

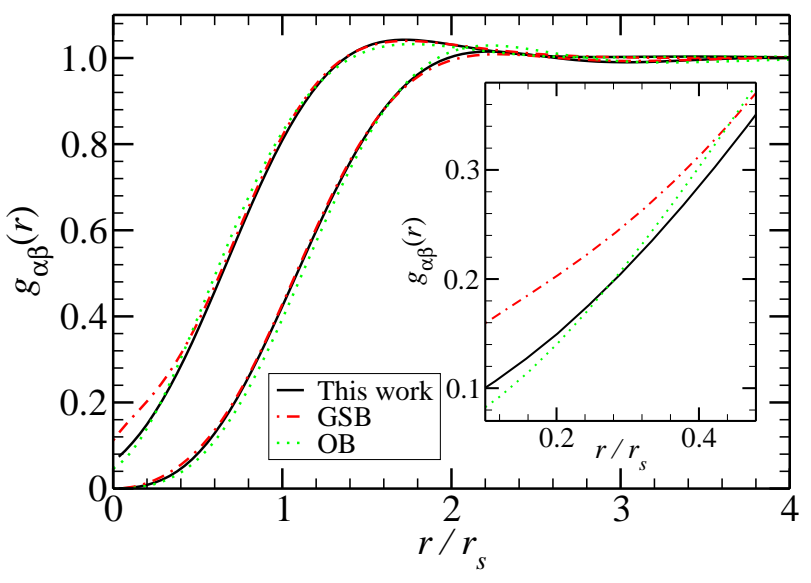

FIG. 5. (Color online) Spin-resolved pair correlation functions (PCFs) for unpolarized HEGs at $r_{\mathrm{s}}=5$ a.u. We show our raw twist-averaged DMC data, together with the fit to DMC results from Ortiz and Ballone ${ }^{20}$ (labeled $\mathrm{OB}$ ) given in their paper, and a fit due to Gori-Giorgi, Sacchetti, and Bachelet ${ }^{23}$ using the DMC data of Ortiz, Harris, and Ballone, ${ }^{22}$ (labeled GSB). The inset shows the low- $r$ region, where the differences are largest.

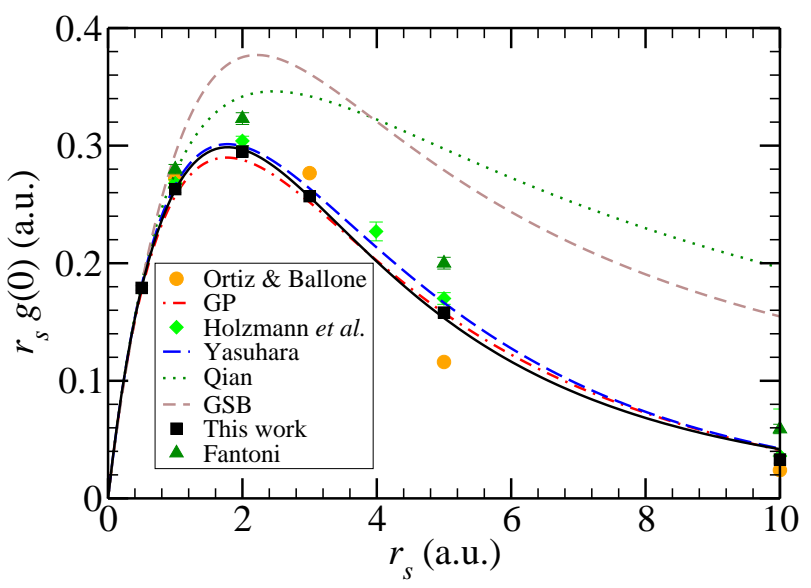

FIG. 6. (Color online) The on-top pair density $g(0)$ (multiplied by $r_{\mathrm{s}}$ ) as a function of $r_{\mathrm{s}}$ for unpolarized systems. The data shown are those of: our averaged DMC and VMC results, together with the fit of Eq. (2) (solid line); Gori-Giorgi and Perdew; ${ }^{13}$ Holzmann et al. $\stackrel{24}{2}$ Yasuhara, $\stackrel{17}{ }$ Qian, ${ }^{19}$ Gori-Giorgi et al. ${ }^{23}$ Ortiz and Ballone, 20 and Fantoni ${ }^{12}$ Error bars are shown for our data, those of Holzmann et al.,${ }^{24}$ and Fantoni, ${ }^{12}$ but they are mostly smaller than the size of the symbols. Our values are slightly smaller than those of Holzmann et al. ${ }^{24}$

used extrapolated estimation due to the small but statistically significant differences between our VMC and DMC results, although these differences are much less pronounced in the antiparallel-spin PCF than in the parallel-minority-spin PCF. This increases the statistical uncertainty in our results, an effect compounded by the increasing sparsity of sampling antiparallel spin coalescences as the polarization increases. Nevertheless, as 
TABLE III. Parameters (in a.u.) obtained from fits to the ontop pair density as a function of $r_{\mathrm{s}}$ using Eq. (2). The data for the unpolarized systems are averaged DMC and VMC results; extrapolated estimation is used for the others.

\begin{tabular}{lllll}
\hline \hline$\zeta$ & \multicolumn{1}{c}{$a$} & $b$ & \multicolumn{1}{c}{$c$} & $d$ \\
\hline 0 & 0.18315 & -0.0784043 & 1.02232 & 0.0837741 \\
0.34 & 0.284118 & -0.110062 & 1.1618 & 0.0874753 \\
0.66 & 0.0659538 & -0.0590569 & 0.836458 & 0.0832258 \\
\hline \hline
\end{tabular}

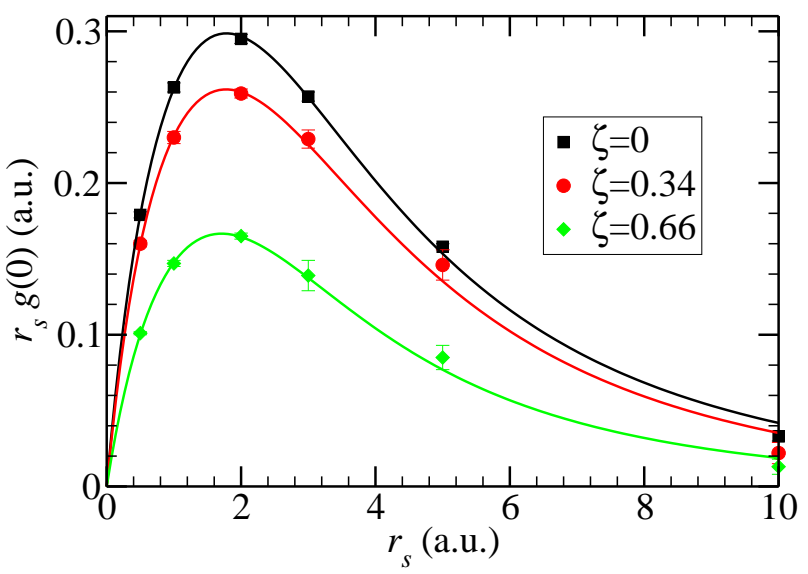

FIG. 7. (Color online) The on-top pair density (multiplied by $r_{\mathrm{s}}$ ) as a function of $r_{\mathrm{s}}$ and $\zeta$. Averaged DMC and VMC data are used for the unpolarized systems, and extrapolated estimates for the polarized systems. Twist averaging was used for all data shown. An error bar is plotted for each data point, although some of them are smaller than the size of the symbols. The partially polarized systems show a larger statistical uncertainty. The lines are fits as discussed in the text.

expected from Fig. 3 the variation of $g(0)$ with $\zeta$ arises largely from changes in the weights in Eq. (1) rather than in the spin-resolved PCFs themselves. We fit our $g(0)$ data to the following parameterized form in the density range $0.5 \leq r_{\mathrm{s}} \leq 20$ a.u.:

$$
g_{\uparrow \downarrow}\left(0 ; r_{\mathrm{s}}\right)=\frac{1+a \sqrt{r_{\mathrm{s}}}+b r_{\mathrm{s}}}{1+c r_{\mathrm{s}}+d r_{\mathrm{s}}^{3}},
$$

and we list the optimal fit parameters obtained in Table III. This functional form obeys the exact high density limit and fits our data well up to $r_{\mathrm{s}}=20$ a.u., although at $r_{\mathrm{s}}=10$ a.u., it tends to give slightly higher values than our QMC calculations obtained, typically by about 2 standard deviations.

\section{B. Static Structure Factor}

Static structure factors (SSFs) with $\zeta=0$ at three representative densities are shown in Fig. 8. The upper curves show $S_{\uparrow \uparrow}-S_{\uparrow \downarrow}$ and the lower curves, $S_{\uparrow \uparrow}+S_{\uparrow \downarrow}$.

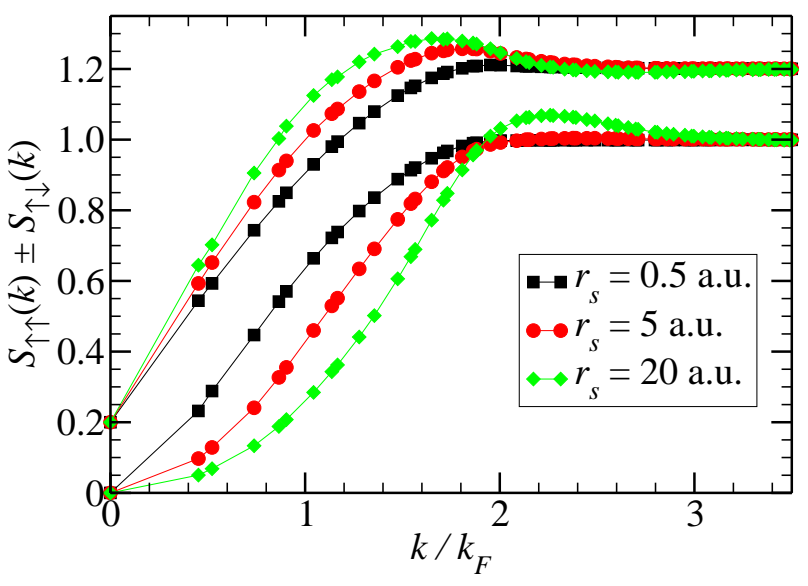

FIG. 8. (Color online) Structure factors at $\zeta=0$ for three densities. The upper curves show $S_{\uparrow \uparrow}-S_{\uparrow \downarrow}$ and are translated upwards by 0.2 units; the lower curves show $S_{\uparrow \uparrow}+S_{\uparrow \downarrow}$. The lines are visual guides only. The statistical uncertainty is smaller than the size of the symbols. The DMC method with twist averaging was used, as the VMC data are again very similar. Intermediate densities lie between the curves shown.

The differences between the DMC and VMC results are again smaller than the statistical noise, and so we plot DMC values. The parallel-spin structure factors change very slowly with density, and the $r_{\mathrm{s}}$-dependence shown arises almost entirely from the antiparallel-spin structure factor, which is (mostly) negative and of increasing magnitude as the density decreases.

Twist averaging was used to obtain the results shown in Fig. 8, and its effect was more pronounced than in the PCFs, as shown in Fig. 9. where we plot antiparallelspin SSFs at $\zeta=0$ with and without twist averaging for the same three densities. Shell-filling effects are clearly visible in the non-twist-averaged data. The VMC and DMC results are very similar and so we can be confident that the differences do not arise from statistical noise. Small finite-size effects are visible in the low- $|\mathbf{k}|$ structure factor data. These correspond to the inevitable finitesize effects in the long-range part of the PCF mentioned above.

\section{Energies}

Twist-averaged DMC energies are reported in TableIV for $r_{\mathrm{s}}=0.5-20 \mathrm{a} . \mathrm{u}$. and for $\zeta=0,0.34,0.66$, and 1 . The data are for finite (small) time steps such that the DMC acceptance probability was almost always greater than $99.7 \%$. We verified in sample cases that the time-step and population-control biases were small: typically less than one part in a thousand of the correlation energy. The finite-size effects in the energy are substantial for the 118-electron HEGs, so we have corrected for these, as discussed in Sec. II] The data are further corrected 


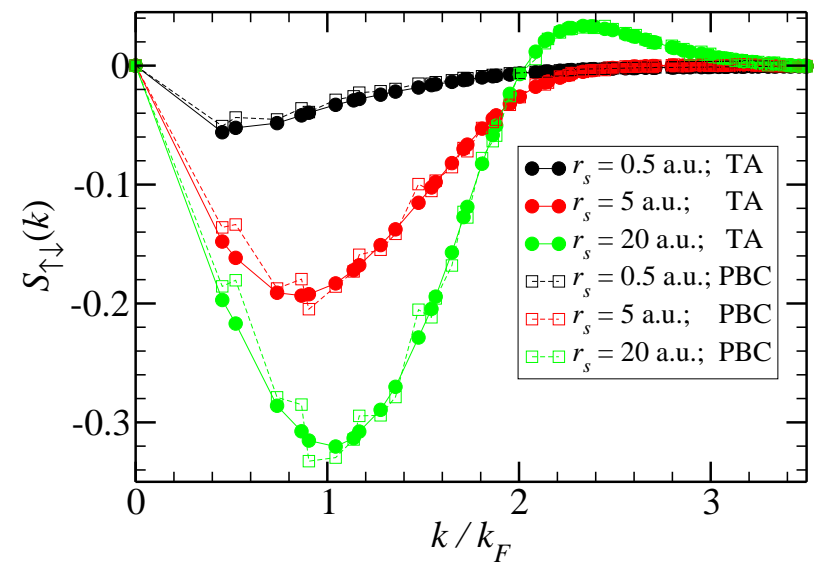

FIG. 9. (Color online) Effect of twist averaging on the antiparallel-spin static structre factor (SSF). We plot SSFs at $\zeta=0$ for three densities, with twist averaging (TA) and without (PBC). Higher densities are towards the top of the graph; intermediate densities lie between the data shown and similar effects are seen in parallel-spin SSFs. Only DMC data are plotted as the VMC data are very similar.

using the control variate method, also discussed in Sec. III because twist-averaging would otherwise lead to large error bars, especially at high densities.

We find that the ground state is unpolarized for all densities studied here, in agreement with the most recent calculations $\stackrel{38}{\underline{30}}$ This is also clearly evident in our VMC results, despite the VMC method suffering a bias towards polarized systems due to the relative ease with which correlation effects in the wave function may be parameterized. This bias decreases with increased variational freedom in the trial wave function.

Our energies for unpolarized and fully polarized HEGs are shown in Fig. 10, where we also plot for comparison the Ceperley-Alder data ${ }^{3}$ and the fit to the latter obtained by Perdew and Zunger,,$\underline{45}$ as used in the LSDA. In addition, a recent high-density (RPA) limit for the polarized system due to Loos and Gill ${ }^{46}$ is shown. The present results follow the Perdew-Zunger fit closely, except at high density, where we find slightly smaller correlation energies, especially at full polarization. The finite-size corrections we have applied are significantly larger than the differences shown in Fig. 10, and it is possible that higher order finite-size corrections would account for the difference in energies obtained. It is worth noting that Ceperley and Alder did not perform QMC calculations at densities higher than $r_{\mathrm{s}}=2$ a.u. for $\zeta=1$ and that the Loos-Gill result also gives smaller correlation energies in this region than the Perdew-Zunger fit.

To compare our intermediate- $\zeta$ energies with those in the literature, we used the procedure developed by Perdew and Zunger to interpolate between $\zeta=0$ and $\zeta=1$. We used our QMC data for the unpolarized and fully polarized systems and interpolated using Eq. (C12) in their paper. These fits are included as part of the
TABLE IV. Energies as a function of density parameter and spin polarization. The DMC data are twist-averaged and include corrections for finite-size effects, as discussed in the text. Time-step extrapolation was not performed. Data include a control variate correction.

\begin{tabular}{lcccc}
\hline \hline \multirow{2}{*}{$r_{\mathrm{s}}$ (a.u.) } & \multicolumn{4}{c}{ Energy $(\mathrm{a} . \mathrm{u} . / \mathrm{elec}$ ) } \\
\hline 0.5 & $3.43011(4)$ & $3.69287(6)$ & $4.44164(6)$ & $5.82498(2)$ \\
1 & $0.58780(1)$ & $0.64919(2)$ & $0.82394(4)$ & $1.14634(2)$ \\
2 & $0.002380(5)$ & $0.016027(6)$ & $0.05475(2)$ & $0.12629(3)$ \\
3 & $-0.067075(4)$ & $-0.061604(5)$ & $-0.04608(2)$ & $-0.017278(4)$ \\
5 & $-0.075881(1)$ & $-0.074208(4)$ & $-0.069548(4)$ & $-0.060717(5)$ \\
10 & $-0.0535116(5)$ & $-0.053214(2)$ & $-0.052375(2)$ & $-0.0507337(5)$ \\
20 & $-0.0317686(5)$ & $-0.0317156(7)$ & $-0.0315940(7)$ & $-0.0313160(4)$ \\
\hline \hline
\end{tabular}

Supplemental Material accompanying this paper $\underline{26}$ The interpolation scheme is very successful at low density, whereas our high-density data for intermediate polarizations are not reproduced with such high accuracy.

In Fig. 11] we show our correlation energies together with a quartic fit in $\zeta$ at each density:

$$
\begin{aligned}
E_{c}\left(r_{\mathrm{s}}, \zeta\right)=f_{0}\left(r_{\mathrm{s}}\right)+\Xi\left(r_{\mathrm{s}}\right) \Delta & f\left(r_{\mathrm{s}}\right) \zeta^{2} \\
+ & {\left[1-\Xi\left(r_{\mathrm{s}}\right)\right] \Delta f\left(r_{\mathrm{s}}\right) \zeta^{4}, }
\end{aligned}
$$

where $\Delta f\left(r_{\mathrm{s}}\right)=f_{1}\left(r_{\mathrm{s}}\right)-f_{0}\left(r_{\mathrm{s}}\right)$ and $f_{\zeta}\left(r_{\mathrm{s}}\right)$ denotes the correlation energy at spin polarization $\zeta$. We fit $f_{\zeta}\left(r_{\mathrm{s}}\right)$ for $\zeta=0$ and 1 over the density range $r_{\mathrm{s}}=0.5-20$ a.u. using the functional form

$$
f_{i}\left(r_{\mathrm{s}}\right)=\frac{\gamma_{i}}{1+\beta_{1}^{i} r_{\mathrm{s}}^{\frac{1}{2}}+\beta_{2}^{i} r_{\mathrm{s}}}
$$

suggested by Ceperley $\stackrel{47}{\leftrightarrows}$ and Perdew and Zunger $\stackrel{45}{w}$ We find the interpolation

$$
\Xi\left(r_{\mathrm{s}}\right)=a+b r_{\mathrm{s}}+c r_{\mathrm{s}}^{2}
$$

gives a good fit to the intermediate- $\zeta$ QMC data. The energies can be extrapolated to the high-density limit using the method of Perdew and Zunger $\stackrel{45}{\underline{n}}$ by matching the correlation energy and potential arising from $f_{i}$ to the high-density expansions at $r_{\mathrm{s}}=0.5$ a.u., although it is not clear if the interpolation between unpolarized and polarized systems used here will be reliable at higher densities.

All data shown in Fig. 111have been corrected for finitesize effects, as discussed above. A control variate correction has also been applied to reduce the statistical uncertainty arising from the finite sample of twist angles, as discussed in Sec. III The optimum parameter values used in the fit of Eqs. (3), (4), and (5) are given in Table $\mathrm{V}$.

\section{Fits to PCFs}

We have performed cubic-spline fits to our PCF data. The spin resolution across the density range $r_{\mathrm{s}}=0.5-20$ 


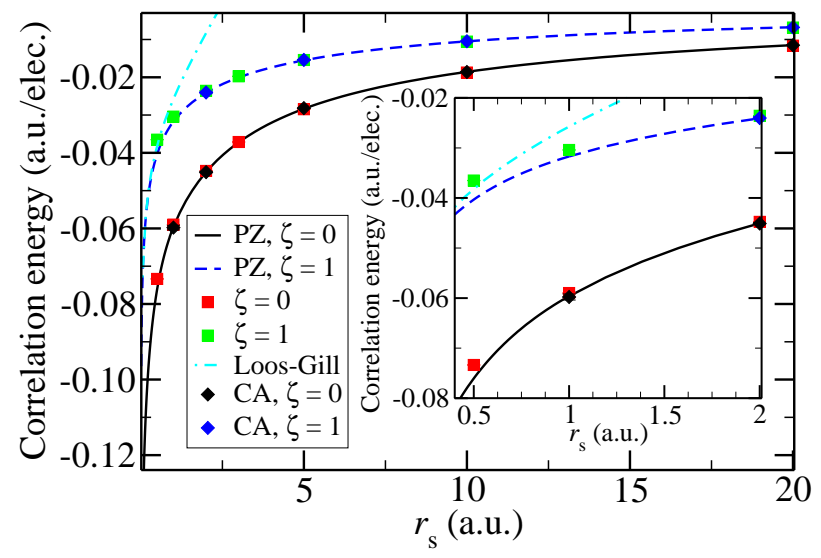

FIG. 10. (Color online) Correlation energy at $\zeta=0$ and 1 against density parameter $r_{\mathrm{s}}$. The present work is simply labeled " $\zeta=0$ " and " $\zeta=1$ ". Also shown are the CeperleyAlder QMC data,$^{3}$ the Perdew-Zunger fit to the latter data $\stackrel{45}{,}$ and the recent high-density result of Loos and Gill ${ }^{46}$ Our data are corrected for finite-size effects. Time step extrapolation was not performed. The statistical error bars are shown, but are usually smaller than the symbols.

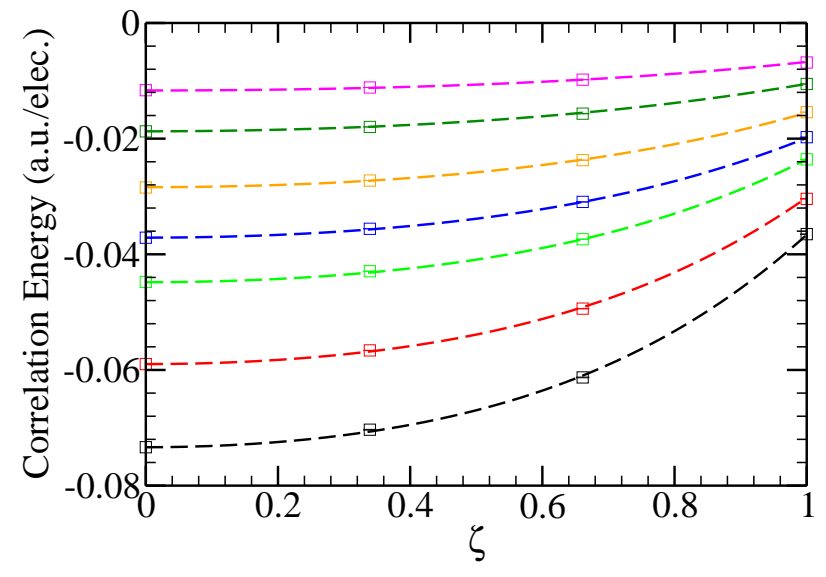

FIG. 11. (Color online) Correlation energies obtained in DMC calculations together with the fit of Eqs. (3), (4), and (5). Dashed lines show the fit; square symbols the QMC data. Lower densities appear towards the top of the graph; higher densities towards the bottom of the figure. From top-tobottom, therefore, the densities shown are: $r_{\mathrm{s}}=20,10,5,3$, 2, 1, and 0.5 a.u. Error bars on the QMC data are shown, but are smaller than the size of the symbols. Corrections have been applied for finite-size effects. Time-step extrapolation was not performed.

a.u. and spin polarizations $\zeta=0,0.34,0.66$, and 1 was well represented by cubic-spline fits with ten knots for each spin-resolved PCF. The boundary conditions used were those of natural splines: second derivatives set to zero at the first and last data points. An example of the fits obtained is given in Fig. [12, where we plot raw QMC data together with fits to the data. The splines reproduce our estimate for the on-top pair density at $r=0$,
TABLE V. Parameters (in a.u.) obtained from the fit to the correlation energy as a function of $r_{\mathrm{s}}$ and $\zeta$ using Eq. (3).

\begin{tabular}{ll}
\hline \hline Parameter & \multicolumn{1}{c}{ Value } \\
\hline$a$ & 0.575073 \\
$b$ & 0.0383567 \\
$c$ & -0.00144917 \\
$\gamma_{0}$ & -0.138971 \\
$\gamma_{1}$ & -0.0633399 \\
$\beta_{1}^{0}$ & 1.04452 \\
$\beta_{2}^{0}$ & 0.311702 \\
$\beta_{1}^{1}$ & 0.872563 \\
$\beta_{2}^{1}$ & 0.225783 \\
\hline \hline
\end{tabular}

and are accurate for $0 \leq r / r_{\mathrm{s}} \leq 3$. For unpolarized and fully polarized systems, we fit the DMC data, whereas for partially polarized systems, we provide fits to the extrapolated estimates of the PCFs. Note that we used down spins as the majority spins in our simulations. We did not impose any exact results as constraints when performing the spline fits, in order to give the most accurate fit possible to our raw data. As discussed above, our QMC data are consistent with the Kimball cusp conditions, and the spline fits follow the data closely in all systems studied. On the other hand, our PCF data inevitably suffer finite-size effects at large- $r$, so various sum rules requiring integration of the PCF over all space will not be satisfied exactly.

These spline fits are available in the form of a small Fortran 90 program that we have written and which is included as part of the Supplemental Material accompanying this article ${ }^{26}$ Subroutines from the SLATEC Common Mathematical Library $\underline{\underline{48}}$ were used to write this program. SSFs can be obtained from these data by Fourier transformation.

We also compared fitting schemes previously used to represent PCF data for these systems, including those

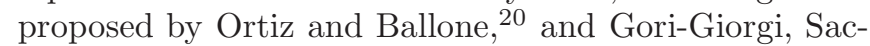
chetti, and Bachelet $\underline{23}$ The former scheme struggled to describe the region around the peak in the PCF with quantitative accuracy. The latter scheme was successful at high density (for $\zeta=0$ ), but less reliable otherwise $\underline{\underline{23}}$

\section{CONCLUSIONS}

We have investigated ground-state properties of the 3D HEG using the VMC and DMC methods. Our simulations cover the density range $r_{\mathrm{s}}=0.5-20 \mathrm{a} . \mathrm{u}$. and spin polarizations $\zeta=0,0.34,0.66$, and 1 . We used highly accurate wave functions incorporating backflow and threebody correlations. Twist averaging was used to reduce finite-size effects, and we show that this can be performed efficiently using a control variate method.

The spin resolution of the PCF and SSF are reported 


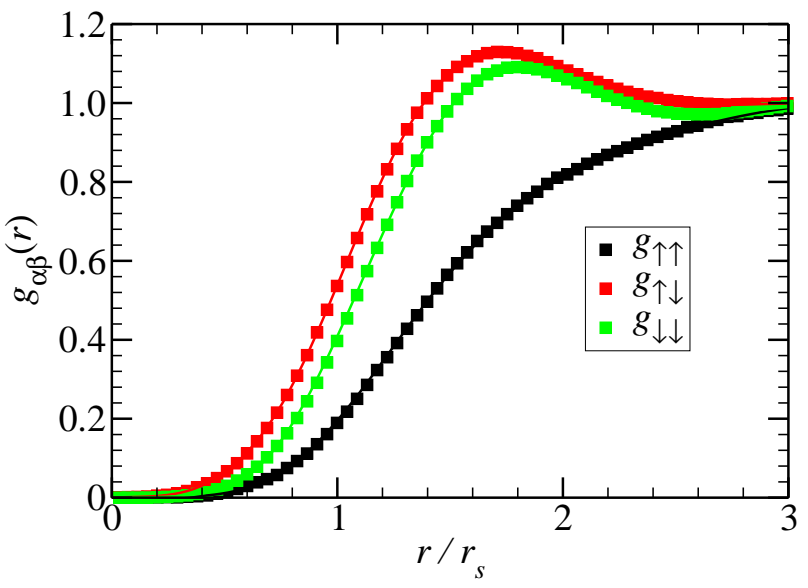

FIG. 12. (Color online) Cubic-spline fits to our twist-averaged extrapolated pair correlation function data at $r_{\mathrm{s}}=20$ a.u. and $\zeta=0.66$. Ten knots were used in the spline fits. Square symbols represent the raw QMC data points and lines represent our spline fits to those data. In all other figures, we have shown the raw QMC data rather than the spline fits displayed here. at each spin polarization studied. At $\zeta=0$, we obtain good agreement with previous QMC studies, except for $r / r_{\mathrm{s}} \lesssim 0.5$, where our data are significantly smaller than those of Gori-Giorgi et al $\stackrel{23}{\underline{2}}$ The effects of twist averaging on these quantities are shown. We report the $r_{\mathrm{s}^{-}}$and $\zeta$-dependence of the energies for 118-electron HEGs. It is hoped that the higher accuracy and lower noise than earlier QMC studies that we have achieved, particularly of the PCFs, will be valuable in, for example, aiding the construction of nonlocal density functionals. A small Fortran 90 program is available to reproduce spline fits to our PCF and SSF data.

\section{ACKNOWLEDGMENTS}

The authors acknowledge financial support from the EPSRC. Computational resources were provided by Cambridge University's High Performance Computing Service. We thank P. López Ríos and A. J. Morris for useful discussions.
${ }^{1}$ G. F. Giuliani and G. Vignale, Quantum Theory of the Electron Liquid, CUP, Cambridge (2005).

2 E. P. Wigner, Phys. Rev. 46, 1002 (1934).

3 D. M. Ceperley and B. J. Alder, Phys. Rev. Lett. 45, 566 (1980).

4 F. H. Zong, C. Lin, and D. M. Ceperley, Phys. Rev. E 66, 036703 (2002).

5 N. D. Drummond, Z. Radnai, J. R. Trail, M. D. Towler, and R. J. Needs, Phys. Rev. B 69, 085116 (2004).

6 J. P. Perdew, Electronic Structure of Solids '91, edited by P. Ziesche and H. Eschrig (Akademie-Verlag, Berlin 1991); J. P. Perdew, K. Burke, and M. Ernzerhof, Phys. Rev. Lett. 77, 3865 (1996); 78, 1396 (1997).

7 O. Gunnarsson, M. Jonson, and B. I. Lundqvist, Phys. Lett. A 59, 177 (1976); Phys. Rev. B 20, 3136 (1979).

8 E. Chacón and P. Tarazona, Phys. Rev. B 37, 4013 (1988).

9 K. Burke, J. P. Perdew, and M. Ernzerhof, J. Chem. Phys. 109, 3760 (1998).

10 J. Toulouse, R. Assaraf, and C. J. Umrigar, J. Chem. Phys. 126, 244112 (2007).

11 R. Assaraf and M. Caffarel, Phys. Rev. Lett. 83, 4682 (1999).

12 R. Fantoni, Solid State Communications 159, 106 (2013).

13 P. Gori-Giorgi and J. P. Perdew, Phys. Rev. B 66, 165118 (2002).

14 P. Gori-Giorgi and J. P. Perdew, Phys. Rev. B 69, 041103(R) (2004).

15 P. Gori-Giorgi and J. P. Perdew, Phys. Rev. B 64, 155102 (2001).

16 A. W. Overhauser, Can. J. Phys. 73, 683 (1995).

17 H. Yasuhara, Solid State Commun. 11, 1481 (1972).

18 I. Nagy, J. I. Juaristi, R. Díez Muiño, and P. M. Echenique, Phys. Rev. B 67, 073102 (2003).

19 Z. Qian, Phys. Rev. B 73, 035106 (2006).

20 G. Ortiz and P. Ballone, Phys. Rev. B 50, 1391 (1994);
Phys. Rev. B 56, 9970 (1997).

21 W. E. Pickett and J. Q. Broughton, Phys. Rev. B 48, 14859 (1993).

22 G. Ortiz, M. Harris, and P. Ballone, Phys. Rev. Lett. 82, 5317 (1999).

23 P. Gori-Giorgi, F. Sacchetti, and G. B. Bachelet, Phys. Rev. B 61, 7353 (2000); Phys. Rev. B 66, 159901(E) (2002).

24 M. Holzmann, B. Bernu, C. Pierleoni, J. McMinis, D. M. Ceperley, V. Olevano, and L. Delle Site, Phys. Rev. Lett. 107, 110402 (2011).

25 S. Paziani, S. Moroni, P. Gori-Giorgi, and G. B. Bachelet, Phys. Rev. B 73, 155111 (2006).

26 See Supplemental Material at [URL] for the Fortran 90 program which evaluates spline fits to our PCF data.

27 R. J. Needs, M. D. Towler, N. D. Drummond, and P. López Ríos, J. Phys.: Condens. Matter 22, 023201 (2010).

28 W. M. C. Foulkes, L. Mitas, R. J. Needs, and G. Rajagopal, Rev. Mod. Phys. 73, 33 (2001).

29 J. B. Anderson, J. Chem. Phys. 65, 4121 (1976).

30 N. D. Drummond, M. D. Towler, and R. J. Needs, Phys. Rev. B 70, 235119 (2004).

31 P. López Ríos, P. Seth, N. D. Drummond, and R. J. Needs, Phys. Rev. E 86, 036703 (2012).

32 Y. Kwon, D. M. Ceperley, and R. M. Martin, Phys. Rev. B 48, 12037 (1993).

33 P. López Ríos, A. Ma, N. D. Drummond, M. D. Towler, and R. J. Needs, Phys. Rev. E 74, 066701 (2006).

34 C. J. Umrigar, K. G. Wilson, and J. W. Wilkins, Phys. Rev. Lett. 60, 1719 (1988).

35 N. D. Drummond and R. J. Needs, Phys. Rev. B 72, 085124 (2005).

36 C. J. Umrigar, J. Toulouse, C. Filippi, S. Sorella, and R. G. Hennig, Phys. Rev. Lett. 98, 110201 (2007).

37 D. M. Ceperley and M. H. Kalos, Monte Carlo Methods in 
Statistical Physics, ed. K. Binder, Springer-Verlag, (1979).

38 C. Lin, F.-H. Zong, and D. M. Ceperley, Phys. Rev. E 64, 016702 (2001).

${ }^{39}$ N. D. Drummond, R. J. Needs, A. Sorouri, and W. M. C. Foulkes, Phys. Rev. B 78, 125106 (2008).

40 S. Chiesa, D. M. Ceperley, R. M. Martin, and M. Holzmann, Phys. Rev. Lett. 97, 076404 (2006).

41 M. Holzmann, B. Bernu, D. M. Ceperley, J. Phys.: Conf. Ser. 321, 012020 (2011).

42 R. Szechtman, Proceedings of the 2003 Winter Simulation Conference, pp 144-149.
43 H. Flyvbjerg and H. G. Petersen, J. Chem. Phys. 91, 461 (1989).

44 H. J. Monkhorst and J. D. Pack, Phys. Rev. B 13, 5188 (1976).

45 J. P. Perdew and A. Zunger, Phys. Rev. B 23, 5048 (1981).

46 P.-F. Loos and P. M. W. Gill, Phys. Rev. B 84, 033103 (2011).

47 D. Ceperley, Phys. Rev. B 18, 31263138 (1978).

48 W. H. Vandevender and K. H. Haskell, ACM SIGNUM Newsletter 17, 16 (1982). 